\title{
Price and Service Quality Competition with Benchmark Effect
}

\author{
Wangyan Xie \\ Jinan University, Guangzhou, China \\ Email: seastar0926@163.com
}

Received 8 January 2016; accepted 22 February 2016; published 25 February 2016

Copyright (C) 2016 by authors and Scientific Research Publishing Inc.

This work is licensed under the Creative Commons Attribution International License (CC BY). http://creativecommons.org/licenses/by/4.0/

c) (i) Open Access

\begin{abstract}
We address the effect of the effect of benchmark effect of industry prominent firm for the influence of the duopoly competition. Consumers always consider prominent firms as the industry benchmark, when they evaluate other firms' product, treating prominent firm as a reference point, thus show the loss aversion behavior. We find that benchmarking effect of the prominent firm makes consumer surplus increase, while decreases both firms' profits.
\end{abstract}

\section{Keywords}

\section{Benchmark Effect, Prominent Firm, Loss Aversion}

\section{Introduction}

When consumers choose brand, they tend to focus on the prominent brand, and treat the prominent firm as reference point. Facing those little-known brands, consumers compare them with prominent firm, that is to say, these well-known brand have a benchmark effect. The prominent firm is probably the first firm to enter the industry, thus can be recognized by consumers earliest, it may also can be the most famous firm in the industry that through marketing and other means. This is illustrated within a duopoly frame by Zhou J. [1] when consumers facing the choice between the prominent firm with its rival, benchmark effect from prominent firm will affect duopoly competition.

In this paper, we study the benchmark effect on competition through the influence of consumer loss aversion behavior [2], that is to say, consumers tend to form their reference point based on the product "characteristics" of prominent firm. Consumer will have a loss or gain when they buy other firms' product or service compared to the prominent firm. Psychology and behavioral science has accumulated a large number of studies that have shown that rely on consumer preferences are often given reference points, and show the loss aversion behavior, that is to say, the degree of pain from a unit of "lost” deep than joy from a unit of "get”. There are also many 
marketing research [3] suggesting that reference point is an important determinant to consumer when they make brand choose, they will compare both firms' "characteristics” and form their utility to make purchase decisions.

Heidhues and Koszegi [4] are the first paper that studies the implications of reference-dependent preferences in duopoly competition. We find that loss aversion is just in price dimension. In this paper, we describe product “characteristics” from price and quality dimension, consumers form the reference point of quality and price.

Overall, we build on the work of Zhou, J. and Karle and Peitz [5], based on empirical evidence of loss aversion on service quality and price [6] and develop a parsimonious model that allows firms to make price and quality decisions in the context of a consumer behavior model when market exits a prominent firm.

The rest of this paper is organized as follows. Section 2 presents the model that is analyzed in Section 3, Section 4 presents the conclusions. All omitted proofs are included in Appendix.

\section{The Model Setup}

We use Hotelling framework (Hotelling) [7] and assume that the market consists of two firms, A and B, each supplying a single distinct brand at a constant unit cost that we normalize to zero. Firm A's located at the endpoint 0 , and firm 2's is at the other endpoint 1 . Firm $A$ is the prominent firm, $t$ is the transportation costs. Firm $A$ provide goods A with price $P_{A}$, quality $S_{A}$, Firm B provide good B with price $P_{B}$, quality $S_{B}$. There is $\alpha$ proportion consumers treat firm $A$ as reference point. Their quality cost is $C=1 / 2 \gamma S^{2}$.

To determine the market demand that the two firms face, let the non-loss-averse consumer type in [0,1], who won't regard firm A to their reference point, they are indifferent between buying good A and good B. be denoted by $\mathrm{X}$, correspondingly, the indifferent, loss-averse consumer is denoted by $\widetilde{X}$.

1) Consumers utility function:

For those non-loss-averse consumers: utility is a standard hoteling model.

$$
\begin{gathered}
U_{A}=V-P_{A}-t x+\theta S_{A} \\
U_{B}=V-P_{B}-t(1-x)+\theta S_{B}
\end{gathered}
$$

For those loss averse consumers: utility = intrinsic utility + psychological utility

Psychologicalutility dependent on the gap between the firm's offer and the benchmark.

$$
\begin{gathered}
U_{A}=V-P_{A}-t x+\theta S_{A} \\
U_{B}=V-P_{B}-t(1-x)+\theta S_{B}-\lambda\left(\theta S_{A}-\theta S_{B}\right)^{+}+\left(\theta S_{B}-\theta S_{A}\right)^{+}+\left(P_{A}-P_{B}\right)^{+}-\lambda\left(P_{B}-P_{A}\right)^{+}
\end{gathered}
$$

$V$ : reservation value; $\theta$ : service factor; $\lambda$ : the weight of loss $(\lambda>1)$.

So, there will are 4 case:

Case $1 S_{A} \geq S_{B}, P_{A} \geq P_{B}$; Case $2 S_{A} \geq S_{B}, P_{A} \leq P_{B}$.

Case $3 S_{A} \leq S_{B}, P_{A} \leq P_{B}$; Case $4 S_{A} \leq S_{B}, P_{A} \geq P_{B}$.

\section{2) The profit of firms}

$$
\begin{gathered}
\Pi_{A}=P_{A}[(1-\alpha) \times X+\alpha \times \tilde{X}]-\frac{1}{2} \gamma_{1} S_{A}^{2} \\
\Pi_{B}=P_{B}[(1-\alpha) \times(1-X)+\alpha \times(1-\tilde{X})]-\frac{1}{2} \gamma_{2} S_{B}^{2}
\end{gathered}
$$

\section{Analysis}

Because it is a multistage game, we use the concept of subgame perfect equilibrium and analyze the decisions of firms and consumers in the reverse order to solve for equilibrium in prices and qualitys. We first focus on $\alpha=0$, which provides us the benchmark against which our results are compared.

\subsection{None Loss Aversion Behavior Consumers ( $\alpha=0)$}

We get an equilibrium solution: 


$$
S_{A}^{0}=\frac{\theta}{3 \gamma}, S_{B}^{0}=\frac{\theta}{3 \gamma}, P_{A}^{0}=t, P_{B}^{0}=t, \Pi_{A}^{0}=\frac{9 t \gamma-\theta^{2}}{18 \gamma}, \Pi_{B}^{0}=\frac{9 t \gamma-\theta^{2}}{18 \gamma}
$$

\subsection{Exit Loss Averse Consumers $(\alpha>0)$}

- For case 1, 2, 3, we get an equilibrium solution, and case 4 have not an equilibrium solution:

\begin{tabular}{ccccc}
\hline & $\alpha=0$ & & $\alpha>0$ & \\
\cline { 3 - 4 } & & case 1 & case 2 & case 3 \\
\hline$S_{A}\left(=S_{B}\right)$ & $\frac{\theta}{3 \gamma}$ & $\frac{\theta(1+\alpha \lambda)}{3 \gamma(1+\alpha)}$ & $\frac{\theta}{3 \gamma}$ & $\frac{\theta(1+\alpha)}{3 \gamma(1+\alpha \lambda)}$ \\
$P_{A}\left(=P_{B}\right)$ & $t$ & $\frac{t}{1+\alpha}$ & $\frac{t}{1+\alpha \lambda}$ & $\frac{t}{1+\alpha \lambda}$ \\
$\Pi_{A}\left(=\Pi_{B}\right)$ & $\frac{9 t \gamma-\theta^{2}}{18 \gamma}$ & $\frac{9 t(1+\alpha) \gamma-\theta^{2}(1+\alpha \lambda)^{2}}{18(1+\alpha)^{2} \gamma}$ & $\frac{9 t \gamma-(1+\alpha \lambda) \theta^{2}}{18(1+\alpha \lambda) \gamma}$ & $\frac{-(1+\alpha)^{2} \theta^{2}+9 t(\gamma+\alpha \gamma \lambda)}{18(1+\alpha \lambda)^{2} \gamma}$ \\
\hline
\end{tabular}

Proposition 1. There exists three Nash Equilibriums, $S_{A}^{3}<S_{A}^{2}<S_{A}^{1}, P_{A}^{1}>P_{A}^{2}=P_{A}^{3} ; \quad S_{B}^{3}<S_{B}^{2}<S_{B}^{1}$, $P_{B}^{1}>P_{B}^{2}=P_{B}^{3}$; in addition, $P_{A}^{i}=P_{B}^{i}, S_{A}^{i}=S_{B}^{i}, \Pi_{A}^{i}=\Pi_{B}^{i} ; \frac{\partial S_{j}^{1}}{\partial \alpha}>0, \frac{\partial S_{j}^{1}}{\partial \lambda}>0, \frac{\partial S_{j}^{3}}{\partial \alpha}<0, \frac{\partial S_{j}^{3}}{\partial \lambda}<0, \frac{\partial P_{j}^{1}}{\partial \alpha}<0$, $\frac{\partial P_{j}^{2}}{\partial \alpha}<0, \frac{\partial P_{j}^{2}}{\partial \lambda}<0, \frac{\partial P_{j}^{3}}{\partial \alpha}<0, \frac{\partial P_{j}^{3}}{\partial \lambda}<0, i=0,1,2,3 ; j=A, B$.

Interpretation: There is no prominent advantage for the prominent firm A. Firm A and firm B make the same service and price decisions, and thus gain the same profits. Firm B, the competitor will follow just his decisions so as to prevent possible utility loss, without additional technology investment/cost.

Proposition 2. $\Pi_{A}^{3}>\Pi_{A}^{2}, \quad \frac{\partial \Pi}{\partial \alpha}<0, \quad \frac{\partial \Pi}{\partial \lambda}<0$, When $\gamma \geq \frac{(2+\alpha+\alpha \lambda) \theta^{2}}{9 t(1+\alpha)(1+\alpha \lambda)}, \quad \Pi_{j}^{1} \geq \Pi_{j}^{3} ; \quad$ When $\gamma \leq \frac{(2+\alpha+\alpha \lambda) \theta^{2}}{9 t(1+\alpha)(1+\alpha \lambda)}, \quad \Pi_{j}^{1} \leq \Pi_{j}^{3}, \quad j=A, B$.

Interpretation: And there is a threshold, where the case 3 can be the better equilibrium than case 1 .

Proposition 2. $\Pi_{A}^{0}>\max \left\{\Pi_{A}^{1}, \Pi_{A}^{3}\right\}, \Pi_{B}^{0}>\max \left\{\Pi_{B}^{1}, \Pi_{B}^{3}\right\}$

Interpretation: loss aversion reduces firm profits and lowers service/product quality as well as prices offered, and increase consumer surplus.

\section{Conclusions}

We study competition on price and quality strategies in markets under benchmark effects of prominent firm. Benchmark is modeled as loss aversion. Consumers form the reference point facing the other firm's goods. We show that the benchmark effects make both firms perform the same, both firms still make the same price/quality decisions, and their profits are the same. The prominent firm can't benefit from the benchmark effect. Since the other firm has motivation to decrease the gap between them. Furthermore, loss aversion has negative impacts in both firms' profit; however, it benefits consumers most of the time.

Because under our setting is that the cost of two firm is the same, so, it will be different when their margin quality cost is asymmetric, it is my next work.

\section{References}

[1] Arbatskaya, M. (2007) Ordered Search. RAND Journal of Economics, 38, 119-126.

[2] Kahneman, D. and Tversky, A. (1979) Prospect Theory: An Analysis of Decision under Risk. Econometrica: Journal of the Econometric Society, 47, 263-291. http://dx.doi.org/10.2307/1914185

[3] Mazumdar, T., Raj, S.P. and Sinha, I. (2005) Reference Price Research: Review and Propositions. Journal of Market- 
ing, 69, 84-102. http://dx.doi.org/10.1509/jmkg.2005.69.4.84

[4] Heidhues, P. and Koszegi, B. (2008) Competition and Price Variation When Consumers Are Loss Averse. American Economic Review, 98, 1245-1268. http://dx.doi.org/10.1257/aer.98.4.1245

[5] Karle, H. and Peitz, M. (2014) Competition under Consumer Loss Aversion. The RAND Journal of Economics, 45, 131. http://dx.doi.org/10.1111/1756-2171.12040

[6] Hardie, B.G.S., Johnson, E.J. and Fader, P.S. (1993) Modeling Loss Aversion and Reference Dependence Effects on Brand Choice. Marketing Science, 12, 378-394. http://dx.doi.org/10.1287/mksc.12.4.378

[7] Hotelling, H. (1929) Stability in Competition. The Economic Journal, 39, 41-57. http://dx.doi.org/10.2307/2224214 


\section{Appendix}

$$
\begin{aligned}
& U_{A}\left(x, P_{A}, S_{A} ; P_{B}, S_{B}\right)=V-P_{A}-t x+\theta S_{A}=U_{B}\left(x, P_{B}, S_{B} ; P_{A}, S_{A}\right)=V-P_{B}-t(1-x)+\theta S_{B} \\
& x=\frac{-P_{A}^{0}+P_{B}^{0}+t+\theta S_{A}^{0}-\theta S_{B}^{0}}{2 t} \\
& \Pi_{A}^{0}\left(P_{A}, S_{A} ; P_{B}, S_{B}\right)=P_{A}^{0}\left[\frac{-P_{A}^{0}+P_{B}^{0}+t+\theta S_{A}^{0}-\theta S_{B}^{0}}{2 t}\right]-\frac{1}{2} \gamma S_{A}^{02} \\
& \frac{\partial \Pi_{A}^{0}}{\partial P_{A}^{0}}=-\frac{P_{A}^{0}}{2 t}+\frac{-P_{A}^{0}+P_{B}^{0}+t+\theta S_{A}^{0}-\theta S_{B}^{0}}{2 t} \\
& \text { Let } \frac{\partial \Pi_{A}^{0}}{\partial P_{A}^{0}}=0, P_{A}^{0}=\frac{1}{2}\left(P_{B}^{0}+t+\theta S_{A}^{0}-\theta S_{B}^{0}\right) \\
& \Pi_{B}^{0}\left(P_{B}, S_{B} ; P_{A}, S_{A}\right)=P_{B}^{0}\left(1-\frac{-P_{A}^{0}+P_{B}^{0}+t+\theta S_{A}^{0}-\theta S_{B}^{0}}{2 t}\right)-\frac{1}{2} \gamma S_{B}^{02} \\
& \frac{\partial \Pi_{B}^{0}}{\partial P_{B}^{0}}=1-\frac{P_{B}^{0}}{2 t}-\frac{-P_{A}^{0}+P_{B}^{0}+t+\theta S_{A}^{0}-\theta S_{B}^{0}}{2 t} \\
& \frac{\partial \Pi_{B}^{0}}{\partial P_{B}^{0}}=0, P_{B}^{0}=\frac{1}{2}\left(P_{A}^{0}+t-\theta S_{A}^{0}+\theta S_{B}^{0}\right) \\
& P_{A}^{0}=\frac{1}{3}\left(3 t+\theta S_{A}^{0}-\theta S_{B}^{0}\right), P_{B}^{0}=\frac{1}{3}\left(3 t-\theta S_{A}^{0}+\theta S_{B}^{0}\right) \\
& \Pi_{A}^{0}\left(P_{A}, S_{A} ; P_{B}, S_{B}\right)=\left[\frac{1}{3}\left(3 t+\theta S_{A}^{0}-\theta S_{B}^{0}\right)\right]\left[\frac{-\left(\frac{1}{3}\left(3 t+\theta S_{A}^{0}-\theta S_{B}^{0}\right)\right)+\left(\frac{1}{3}\left(3 t-\theta S_{A}^{0}+\theta S_{B}^{0}\right)\right)+t+\theta S_{A}^{0}-\theta S_{B}^{0}}{2 t}\right] \\
& -\frac{1}{2} \gamma S_{A}^{02} \\
& \frac{\partial \Pi_{A}^{0}}{\partial S_{A}^{0}}=-S_{A}^{0} \gamma+\frac{\theta\left(3 t+\theta S_{A}^{0}-\theta S_{B}^{0}\right)}{18 t}+\frac{\theta\left[2 t+\frac{2 \theta S_{A}^{0}}{3}-\frac{2 \theta S_{B}^{0}}{3}+\frac{1}{3}\left(-3 t-\theta S_{A}^{0}+\theta S_{B}^{0}\right)\right]}{6 t} \\
& \text { Let } \frac{\partial \Pi_{A}^{0}}{\partial S_{A}^{0}}=0, S_{A}^{0}=\frac{3 t \theta-S_{B}^{0} \theta^{2}}{9 t \gamma-\theta^{2}} \\
& \Pi_{B}^{0}\left(P_{B}, S_{B} ; P_{A}, S_{A}\right)=\left[\frac{1}{3}\left(3 t-\theta S_{A}^{0}+\theta S_{B}^{0}\right)\right]\left[1-\frac{-\left[\frac{1}{3}\left(3 t+\theta S_{A}^{0}-\theta S_{B}^{0}\right)\right]+\frac{1}{3}\left(3 t-\theta S_{A}^{0}+\theta S_{B}^{0}\right)+t+\theta S_{A}^{0}-\theta S_{B}^{0}}{2 t}\right] \\
& -\frac{1}{2} \gamma S_{B}^{02} \\
& \frac{\partial \Pi_{B}^{0}}{\partial S_{B}^{0}}=-S_{B}^{0} \gamma+\frac{\theta\left(3 t-\theta S_{A}^{0}+\theta S_{B}^{0}\right)}{18 t}+\frac{1}{3} \theta\left[1-\frac{2 t+\frac{2 \theta S_{A}^{0}}{3}-\frac{2 \theta S_{B}^{0}}{3}+\frac{1}{3}\left(-3 t-\theta S_{A}^{0}+\theta S_{B}^{0}\right)}{2 t}\right]
\end{aligned}
$$




$$
\frac{\partial \Pi_{B}^{0}}{\partial S_{B}^{0}}=0, \quad S_{B}^{0}=\frac{3 t \theta-S_{A}^{0} \theta^{2}}{9 t \gamma-\theta^{2}}
$$

$S_{A}^{0}=\frac{\theta}{3 \gamma}, \quad S_{B}^{0}=\frac{\theta}{3 \gamma}, \quad P_{A}^{0}=t, \quad P_{B}^{0}=t, \quad \Pi_{A}^{0}=\frac{9 t \gamma-\theta^{2}}{18 \gamma}-c, \quad \Pi_{B}^{0}=\frac{9 t \gamma-\theta^{2}}{18 \gamma}$

Then, we can get the other cases' equilibrium solutions. 